\title{
A LIBRATION MODEL FOR ENCELADUS BASED ON GEODETIC CONTROL POINT NETWORK ANALYSIS
}

\author{
Nadezhdina I.E. ${ }^{\text {a }}$, Zubarev A.E. ${ }^{\text {a }}$, Brusnikin E.S. ${ }^{\text {a }}$ and Oberst J. ${ }^{\text {a, b, c }}$ \\ ${ }^{a}$ Moscow State University of Geodesy and Cartography (MIIGAiK), MIIGAiK Extraterrestrial Laboratory (MexLab), \\ Gorokhovskiy pereulok, 4, Moscow, 105064, Russia - i nadejdina@ miigaik.ru \\ ${ }^{\mathrm{b}}$ German Aerospace Center (DLR) \\ ${ }^{\mathrm{c}}$ Technical University Berlin, Berlin, Germany
}

Commission IV, WG IV/8

KEY WORDS: Enceladus, photogrammetry image processing, 3D control point network, estimation of shape and forced libration

\begin{abstract}
:
A new global control point network was derived for Enceladus, based on Cassini and Voyager-2 image data. Cassini images were taken from 2005 to 2014, for Voyager we have only one flyby in the middle of 1981. We have derived 3D Cartesian coordinates for 1128 control points as well as improved pointing data for 12 Voyager and 193 Cassini images in the Enceladus-fixed coordinate system. The point accuracies vary from $55 \mathrm{~m}$ to $2900 \mathrm{~m}$ (average point accuracy $-221 \mathrm{~m}$ ). From tracking of the control points we detect a librational motion described by a model which includes 3 different periods and amplitudes (Rambaux et al., 2011). We determine the amplitudes for each term. Our new control point network has a higher number of point measurements and a higher accuracy than previous data (Giese et al., 2014).
\end{abstract}

\section{INTRODUCTION}

Enceladus, a satellite of Saturn, is covered with ice and reflects almost all incoming sunlight. The possible presence of an ocean and ongoing ejection of vapour from it through cracks in its surface ice (Perrya et al., 2015) shell makes the satellite a promising candidate for search for microbial life in the Solar System (Tobie et al., 2014). Therefore imaging of Enceladus takes a significant share in the operation program of Cassini mission to the Saturn system: since 2004 a lot of remote sensing data relevant to Enceladus surface processes have been acquired. Using different filters, Cassini cameras obtain images in several spectral channels (Knowles, 2012). In the frame of this research new spectral images were used to refine the global three-dimensional control point network of Enceladus.

The coordinates of the surface points are determined from the measurements of their line/sample coordinates in large numbers of overlapping images ("image blocks") by iterative inversion techniques, in photogrammetry called "bundle block adjustments". An important product of the bundle block adjustments is the corrected spacecraft position and pointing data for all images that are involved. Hence, the establishment of control point networks is essential for the production of maps, accurately positioned in the body-fixed reference frame. Such accurate maps, in turn, are essential for spacecraft navigation or operation of onboard instruments, e.g., if a camera is to be pointed at a specific target of interest.

Dense clouds of 3D control point networks are an essential framework for shape studies. Another important application of control point analysis is the tracking of the points over time, from which unknown rotational parameters of the planetary body (e.g., rotational axis orientation or librational motion) may be determined. Control points can also be used as reference markers for positional measurements of the target body against the star background. The renovated network was used to recalculate the fundamental parameters of the Saturn satellite: Enceladus shape model and the forced libration.

\section{ENCELADUS LIBRATION MODEL}

The libration amplitude $\gamma$ predicted for Enceladus was described as (Rambaux, et al., 2011):

$$
\gamma=\sum_{i} \frac{\omega_{0}^{2} H_{i}}{\omega_{0}^{2}-\omega_{i}^{2}} \sin \left(\omega_{i} t+\alpha_{i}\right)
$$

where $\omega_{0}=n \sqrt{\frac{3(B-A)}{C}}$ is the free libration frequency ( $n$ is mean motion), $t$ is Ephemeris time, and $\omega_{i}, H_{i}, \alpha_{i}$ are parameters given in Table 1. The libration amplitude for each term can be calculated as:

$$
A_{i}=\frac{\omega_{0}^{2}}{\omega_{0}^{2}-\omega_{i}^{2}} H_{i}
$$

\begin{tabular}{|c|c|c|c|}
\hline $\boldsymbol{i}$ & $\begin{array}{c}\text { Period } \\
2 \pi / \omega_{i}, \\
\text { days }\end{array}$ & $\begin{array}{c}\text { Magnitude } \\
H_{i}, \\
\text { arcsec }\end{array}$ & $\begin{array}{c}\text { Phase } \\
\alpha_{i},\end{array}$ \\
\hline 1 (forced by Saturn) & 1.371983 & 1954.84 & 10.69 \\
\hline 2 (forced by Dione) & 4035.64 & 933.30 & 73.81 \\
\hline 3 (forced by Dione) & 1418.93 & 676.54 & -43.01 \\
\hline
\end{tabular}

Table 1. Libration parameters used in the calculations

(Rambaux et al., 2011).

\section{IMAGE DATA AND CONTROL POINT ANALYSIS}

The Cassini spacecraft is equipped with two frame camera systems, the NAC (Narrow Angle Camera, $\mathrm{f}=2003.44 \mathrm{~mm}$ ) and the WAC (Wide Angle Camera, $\mathrm{f}=200.77 \mathrm{~mm}$ ) with identical sensor arrays $(1024 \times 1024$ pixels, pixel size: $12.0 \mu \mathrm{m})$ (Knowles, 2012), which makes the cameras very suitable for geodetic work. In contrast, the Voyager-2 spacecraft, which made one Enceladus flyby in 1981, was equipped with two Vidicon sensors, NAC $\mathrm{f}=1500.190 \mathrm{~mm}$ (pixel size: $11.789 \mu \mathrm{m}$ ) and WAC f=200.465 mm (pixel size: $11.800 \mu \mathrm{m}$ ) (Benesh and Jepsen, 1978). Unfortunately, these Vidicon images suffer from 
significant geometric distortions and require sophisticated geometric calibration schemes, e.g., implemented in the planetary image processing package VICAR (Deen, 2015).

Typically, control point measurements on Cassini and Voyager2 images were combined in the block adjustment to warrant a contiguous image block. 12 Voyager-2 (Voyager PDS, 2008) and 193 (3 WAC and 190 NAC) Cassini images (Cassini PDS, 2016) were selected. Using recently updated navigation information delivered in the form of SPICE-kernels (Voyager SPICE, 2015; Cassini SPICE, 2016) image exterior and interior orientation parameters were determined. The pixel resolution ranges from $20 \mathrm{~m} /$ pixel to $1080 \mathrm{~m} /$ pixel (average $210 \mathrm{~m} /$ pixel) for Cassini images and from $853 \mathrm{~m} /$ pixel to $2390 \mathrm{~m} /$ pixel for Voyager-2 images (average $1341 \mathrm{~m} / \mathrm{pixel}$ ). Following the technique implemented for Ganymede image processing (Zubarev et al., 2015) based on special developed iterative method (Zubarev et al., 2016a) we computed a global 3D control point network (CPN) for Enceladus and solved for a rotation model, including rotation axis orientation and libration.

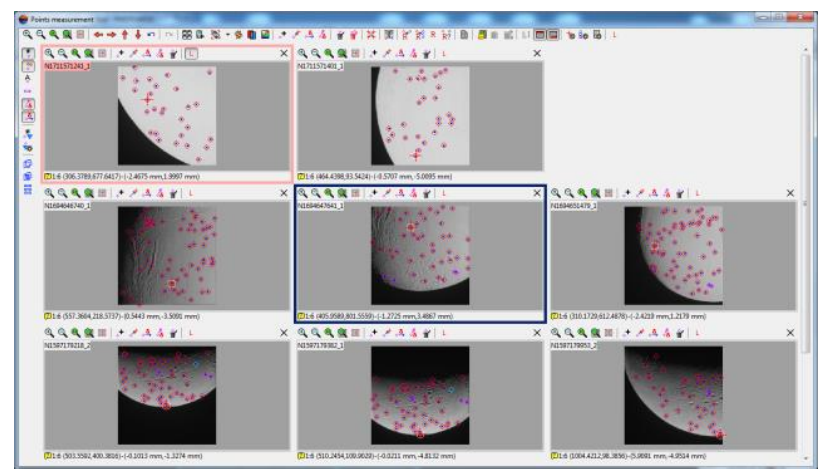

Figure 1. Tie-point measurements using the photogrammetric software package PHOTOMOD ${ }^{\mathrm{TM}}$

A total of 1128 points were observed 14121 times with a minimum of 3 observations and a maximum of 36 observations per point in the image data set (Fig. 1). The average number of observations per point is 12 . An a-priori uncertainty of 1 pixel for the image coordinate observations was assumed. Point measurements and bundle block adjustments were carried out by means of the software package PHOTOMOD (Adrov et. al., 1995), the current version 6.0 (PHOTOMOD, 2016). The package was adapted to create global control point networks and extended by specially developed software modules (Zubarev et al., 2016b, this issue) that provide additional functionalities for planetary image processing.

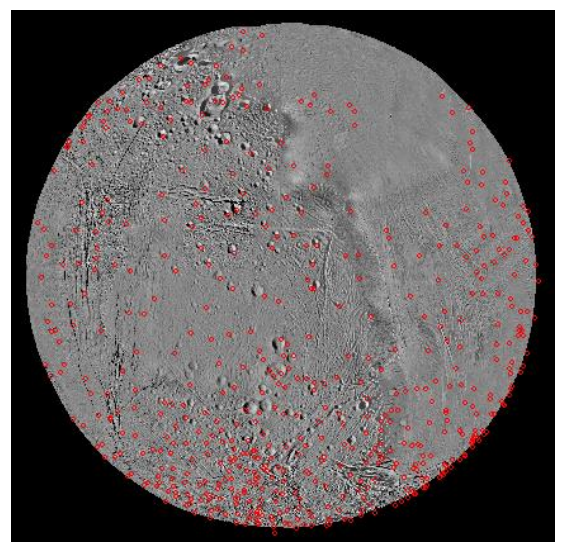

Figure 2. New 3D control point network of Enceladus

\section{RESULTS}

Following the bundle block adjustment, we obtain 3D coordinates of control points (Fig.2) as well as improved data for spacecraft position and camera pointing. The adjustment yields Cartesian coordinates of CPN including their errors. The control points are rather evenly distributed over the surface of Enceladus (Fig. 3). However, errors of the coordinates vary owing to limited numbers of images and limited image resolution for some areas. The area where we use Voyager-2 images for gap-fill is poorly controlled.

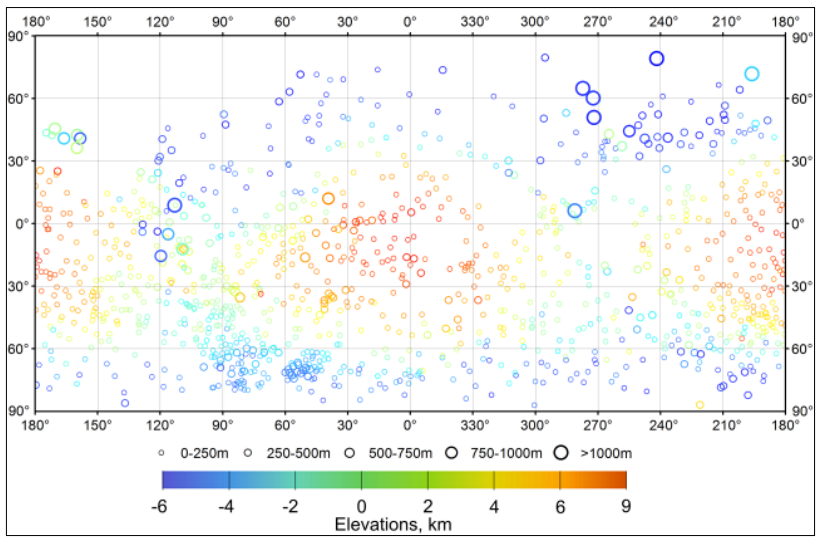

Figure 3. Distribution of Enceladus control points. The size of the circles represents the accuracy of the each point. Color represents the elevations in meters above the best-fit sphere with radius $252.0 \mathrm{~km}$

Using 3D coordinates of the control points we determined new shape parameters of Enceladus (Table 2).

\begin{tabular}{|c|c|c|c|}
\hline Body & $\mathrm{a}, \mathrm{km}$ & $\mathrm{b}, \mathrm{km}$ & $\mathrm{c}, \mathrm{km}$ \\
\hline$[$ Thomas, 2015] & $256.2 \pm 0.3$ & $251.4 \pm 0.2$ & $248.6 \pm 0.2$ \\
\hline Sphere & \multicolumn{3}{|c|}{$251.93 \pm 0.08$} \\
\hline Spheroid & \multicolumn{2}{|c|}{$253.74 \pm 0.07$} & $248.04 \pm 0.12$ \\
\hline Ellipsoid & $255.98 \pm 0.08$ & $251.33 \pm 0.08$ & $248.08 \pm 0.08$ \\
\hline
\end{tabular}

Table 2. Enceladus global shape parameters, derived from new control point network

Based on gravity measurements made by Cassini, McKinnon (2015) found that Enceladus is not in hydrostatic equilibrium. This is also confirmed by the ratio of $(\mathrm{b}-\mathrm{c}) /(\mathrm{a}-\mathrm{c})=0.41$, obtained in our study, far from the hydrostatic equilibrium ratio of 0.25 . In other research, Iess et al. (2014) suggested that the core is in hydrostatic equilibrium, and that an ice layer is in the isostatic. A more accurate model of the internal structure of Enceladus, consisting of a core, water and ice layers (with thickness decreasing towards the poles), was proposed by McKinnon (2015). In another work (Thomas et al., 2015) was shown that it is possible to calculate the thickness of the shell using the measured libration of Enceladus.

To support these studies of Enceladus internal structure we computed an improved libration model (Table 3), using our method based on control points (Nadezhdina and Zubarev, 2014). In the first step we use the nominal libration model (Rambaux et al., 2011), which includes 3 terms with different periods (Table 1). This step gives us the errors in spacecraft positions after adjustment. In the second step we vary the magnitude $H_{i}$ for the first term while keeping the other two fixed, and find the magnitude where we have minimum RMS 
errors in spacecraft positions after the adjustment. In the third step we do the same operation, but we fix the first magnitude determined in the second step and third term. So, after the third step we obtain the magnitude for the second term. In the last step we fix obtained magnitudes for the first 2 terms in order to determine the third value. As a result, we derived libration amplitudes as minimum errors in spacecraft positions for the dataset (Fig. 4).

\begin{tabular}{|c|c|c|c|}
\hline $\mathrm{i}$ & $\begin{array}{c}\text { Period } \\
2 \pi / \omega_{i}, \\
\text { days }\end{array}$ & $\begin{array}{c}\text { Amplitude } A_{i}, \\
\operatorname{arcsec}\end{array}$ & $\begin{array}{c}\text { Magnitude } H_{i}, \\
\text { arcsec }\end{array}$ \\
\hline 1 & 1.371983 & 560 & -10540 \\
\hline 2 & 4035.64 & 1100 & 1100 \\
\hline 3 & 1418.93 & 420 & 420 \\
\hline
\end{tabular}

Table 3. The parameters of the improved libration model

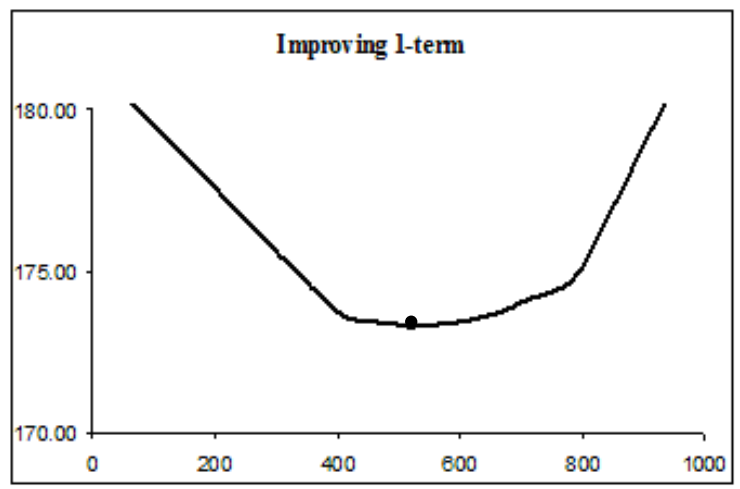

a)

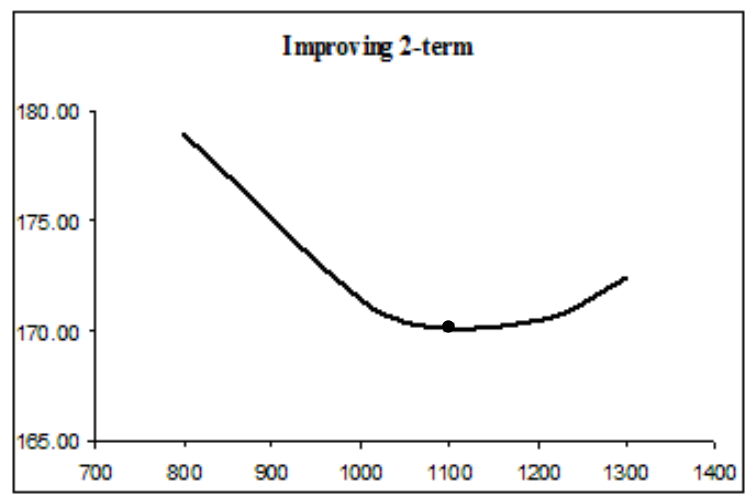

b)

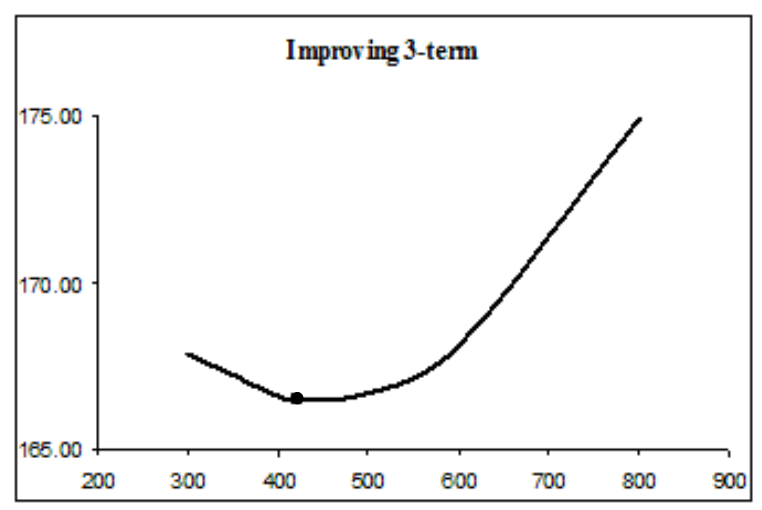

c)

Figure 4. Dependence of libration amplitudes (horizontal axis) and errors in spacecraft positions (vertical axis) for each term: minimum errors (black points on the curves) indicate the measured amplitude of libration
The error functions are very smooth, implying that the function minimum and libration amplitude cannot be detected more precisely than $\pm 50-100$ arc seconds $\left( \pm 0.014^{\circ}-0.028^{\circ}\right)$. The average object point accuracies $\sigma_{\mathrm{x}}, \sigma_{\mathrm{y}}, \sigma_{\mathrm{z}}$ of $150 \mathrm{~m}, 121 \mathrm{~m}, 88 \mathrm{~m}$ respectively (Table 4) were computed for control points measured both in Cassini and Voyager-2 images and include improvement of libration motion.

\begin{tabular}{|l|c|c|c|}
\hline \multicolumn{1}{|c|}{ Errors } & Average & Min & Max \\
\hline$\sigma_{\mathrm{x}}, \mathrm{km}$ & 0.15 & 0.04 & 2.18 \\
\hline$\sigma_{\mathrm{y}}, \mathrm{km}$ & 0.12 & 0.03 & 1.91 \\
\hline$\sigma_{\mathrm{z}}, \mathrm{km}$ & 0.09 & 0.02 & 0.90 \\
\hline$\sigma_{\mathrm{xyz}}, \mathrm{km}$ & 0.22 & 0.06 & 2.90 \\
\hline
\end{tabular}

Table 4. Point accuracies with libration motion improvements

\section{CONCLUSION}

We computed a new geodetic 3D control point network for Enceladus using all available image data. Base on control points libration amplitude was determined for all terms in the predicted model described by Rambaux et al. (2011). We showed that the predicted librations forced by Dione (2nd and 3rd terms) do not correspond to our adjustment results. Forced libration of Enceladus measured in our study (Fig. 5) is in agreement with the model obtained by Giese et al. (2011).

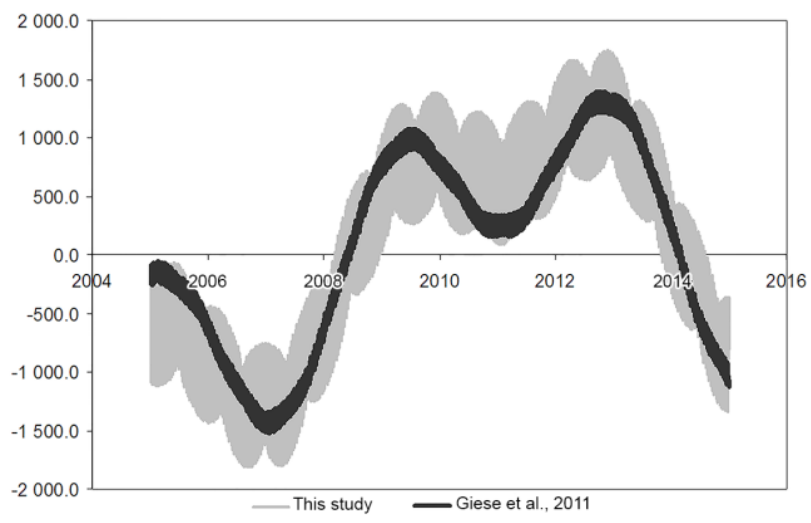

Figure 5. Forced libration of Enceladus: vertical axis is amplitude of the libration in arc seconds; horizontal axis represents the Cassini mission timeline

To determine the accurate libration amplitude of each term, observations for at least for one full period are needed. Available observations made from 2005 to 2012 provide estimations for two of the libration amplitude terms: 1.37 days (Fig.4a) and 3.88 years (Fig.4c). However, the long-period libration amplitude $\sim 11$ years (Fig.4b) is not fully covered by the observations, so it is therefore poorly constrained and requires improvement. In comparison with other recent studies, the short-period of libration $0.120^{\circ} \pm 0.014$ (Thomas et al., 2015) is closer to our results of $0.155^{\circ} \pm 0.014$ than the value of $-0.029^{\circ}$ (Rambaux et al., 2011).

We suggest that our measurements (Table 3) provide new possibilities to receive additional information about internal structure of Enceladus based on model proposed by McKinnon (2015) and help to estimate more accurately thickness of the shell using the improved libration amplitude. 


\section{ACKNOWLEDGEMENTS}

This work was carried out in MIIGAiK and supported by Russian Science Foundation, project \#14-22-00197.

\section{REFERENCES}

Acton C.H. Ancillary data services of NASA's navigation and ancillary information facility 1996. Planetary Space Science, 44 (1), pp. 65-70.

Adrov V.N, Chekurin A. D, Sechin A. Yu, Smirnov A. N, Adam-Guillaume J.-P, Quessete J.-P. 1995, Program PHOTOMOD: digital photogrammetry and stereoscopic image synthesis on a personal computer. Digital Photogrammetry and Remote Sensing. Ed.: Fedosov E.A. Proc. SPIE, 2646, pp. 89 96. DOI: $10.1117 / 12.227853$.

Benesh M. and Jepsen P. Voyager. Imaging Science Subsystem, Calibration report. 1978, Jet Propulsion Laboratory. http://pdsrings.seti.org/voyager/iss/Benesh_Jepsen_1978.pdf.

Cassini PDS, 2016: http://pdsimaging.jpl.nasa.gov/volumes/iss.html.

Cassini SPICE, 2016: http://naif.jpl.nasa.gov/pub/naif/pds/data

Deen R. VICAR: Quick-Start Guide. 2015, California Institute of Technology. http://wwwmipl.jpl.nasa.gov/vicar_os/v1.0/vicar-

docs/VICAR guide_1.0.pdf.

Giese B. An upper limit on Enceladus' obliquity. 2014, EPSC Abstracts, $\quad$ Vol. $9, \quad$ EPSC2014-419, http://meetingorganizer.copernicus.org/EPSC2014/EPSC2014$\underline{419 . p d f}$

Giese B., Hussmann H., Roatsch T., Helfenstein P., Thomas P.C., and Neukum G. EPSC Abstracts, Vol. 6, EPSC-DPS2011976, 2011.

Iess L., Stevenson D.J., Parisi M., Hemingway D., Jacobson R.A., Lunine J.I., Nimmo F., Armstrong J.W., Asmar S.W., Ducci M., Tortora P. The gravity field and interior structure of Enceladus. 2014, Science, 344, pp. 78-80.

Knowles B. Cassini Imaging Science Subsystem (ISS) Data User's Guide. 2012, Space Science Institute. http://pdsrings.seti.org/cassini/iss/ISS Data User Guide 120703.pdf.

McKinnon, W.B. Effect of Enceladus' rapid synchronous spin on interpretation of Cassini gravity. 2015, Geophys. Res. Lett. 42. http://dx.doi.org/10.1002/2015GL063384.

Nadezhdina I., Patraty V., Shishkina L., Zhukov D., Zubarev A., Karachevtseva I., Oberst J. Global shape estimates and GIS cartography of Io and Enceladus using new control point network. Geophysical Research Abstracts, EGU General Assembly, Vienna, Austria 22-27 April 2012, Vol. 14, EGU2012-11210.

http://meetingorganizer.copernicus.org/EGU2012/EGU2012$\underline{11210 . p d f}$

Nadezhdina I.E., Zubarev A.E. Formation of a reference coordinate network as a basis for studying the physical parameters of Phobos. 2014, Solar System Research, Vol. 48, No. 4, pp. 269-278. DOI: 10.1134/S003809461404008X.
Perry M.E. , Teolis B.D., Hurley D.M., Magee B.A., Waite J.H., Brockwell T.G., Perryman R.S., McNutt Jr. R.L. Cassini INMS measurements of Enceladus plume density. 2015, Icarus 257, pp. 139-162. DOI: doi:10.1016/j.icarus.2015.04.037.

\section{PHOTOMOD, 2016: www.racurs.ru/?lng=eng\&page=634}

Rambaux N., Van Hoolst T., Karatekin. O. Libration response of Europa, Ganymede, and Callisto with an ocean for a nonKeplerian orbits. 2011, Astronomy \& Astrophysics 527, A118. DOI: http://dx.doi.org/10.1051/0004-6361/201015304. http://www.aanda.org/articles/aa/pdf/2011/03/aa15304-10.pdf.

Thomas P.C., Tajeddine R., Tiscareno M.S., Burns J.A., Joseph J., Loredo T.J., Helfenstein P., Porco C. Enceladus's measured physical libration requires a global subsurface ocean. 2015, Icarus, 264 (2016) 37-47.

Tobie G., Teanby N. A., Coustenis A., Jaumann R., Raulin F., Schmidt J., Carrasco N., Coates A. J., Cordier D., De Kok R., Geppert W. D., Lebreton J.-P., Lefevre A., Livengood T. A., Mandt K. E., Mitri G., Nimmo F., Nixon C. A., Norman L., Pappalardo R. T., Postberg F., Rodriguez S., Schulze-Makuch D., Soderblom J. M., Solomonidou A., Stephan K., Stofan E. R.,Turtle E. P., Wagner R. J., West R. A., Westlake J. H.. Science goals and mission concept for the future exploration of Titan and Enceladus. 2014, Planetary and Space Science, Volume 104, pp. 59-77. DOI: 10.1016/j.pss.2014.10.002

VICAR, 2015: http://www-mipl.jpl.nasa.gov/external/vicar.html Voyager PDS, 2008: $\underline{\underline{t t p}: / / \mathrm{pds}-}$ imaging.jpl.nasa.gov/volumes/voyager.html.

Voyager $\quad$ SPICE,
http://naif.jpl.nasa.gov/pub/naif/VOYAGER/kernels.

Zubarev A.E., Nadezhdina I., Oberst J., Hussmann H., Stark A. New Ganymede control point network and global shape model. 2015, Planetary and Space Science. Volume 117, pp. 246-249. DOI:10.1016/j.pss.2015.06.022.

Zubarev A.E., Nadezhdina I.E., Brusnikin E.S., Karachevtseva I.P. and Oberst J. Methodology of image processing using the inhomogeneies data for estimation of basic parameters of celestial bodies (the example of Ganymede). 2016a, Solar System Research, No. 5 (in press).

Zubarev A.E., Nadezhdina I.E., Kozlova N.A., Brusnikin E.S., Karachevtseva I.P. Special software for planetary image processing and research. 2016b, The International Archives of the Photogrammetry, Remote Sensing and Spatial Information Sciences (this issue). 